\title{
Key-interventions derived from three evidence based guidelines for management and follow-up of patients with HFE haemochromatosis
}

Annick Vanclooster ${ }^{1 *}$, Hub Wollersheim ${ }^{2}$, Kris Vanhaecht ${ }^{3}$, Dorine Swinkels ${ }^{4}$, Bert Aertgeerts ${ }^{5}$, David Cassiman ${ }^{1}$ and on behalf of the Haemochromatosis working group

\begin{abstract}
Background: HFE-related hereditary haemochromatosis $(\mathrm{HH})$ is a common autosomal recessive disorder with clinical manifestations ranging from asymptomatic disease to possible life-threatening complications. Cirrhosis, hepatocellular carcinoma, diabetes mellitus or osteoporosis can develop in $\mathrm{HH}$ patients not treated or monitored optimally. The purpose of this study was to develop key-interventions (KI's) to measure and improve the quality of care delivered to patients diagnosed with $\mathrm{HH}$.

Methods: A RAND-Modified Delphi method was used to develop KI's. In the first round of a scoring form to prioritize the recommendations extracted from evidence-based guidelines was circulated between experts. The results of this survey were discussed in a consensus meeting, followed by a final appraisal of the selected recommendations. This resulted in a list of measurable Kl's.

Results: Initially, 41 key recommendations on screening, diagnosis and treatment/management were extracted from three existing guidelines on $\mathrm{HH}$ (European Association for the Study of the Liver, American Association for the Study of Liver Diseases and Dutch guideline on HH). Finally, a core set of 24 recommendations resulted in 15 Kl's.
\end{abstract}

Conclusions: This manuscript presents the results of the process to develop Kl's to measure and improve the quality of care for patients with $\mathrm{HH}$.

Keywords: Hereditary haemochromatosis, Key-interventions, Recommendations, Consensus

\section{Background}

HFE-related hereditary haemochromatosis $(\mathrm{HH})$ is a common chronic autosomal recessive disorder, with a genetic prevalence of $1 / 200$ to $1 / 400$. It has an estimated carrier frequency of $1 / 10$ in those from Northern European descent. The phenotype results from inappropriate accumulation of iron, resulting in end-organ damage [1]. Symptoms can be absent, but complications may also be debilitating or even fatal such as diabetes mellitus, osteoporosis, cirrhosis or hepatocellular carcinoma [2-4]. Patients with $\mathrm{HH}$ are seen, due to the variety of symptoms, by many

\footnotetext{
* Correspondence: Annick.vanclooster@uzleuven.be

'Department of Hepatology and Metabolic Center, University Hospital

Gasthuisberg, Herestraat 49, 3000 Leuven, Belgium

Full list of author information is available at the end of the article
}

different professionals, ranging from hepatogastroenterologists, hematologists, rheumatologists, general practitioners to nurses. The varying published criteria for case definition, referral, diagnosis, interpretation of test results, follow-up and family screening approaches may lead to confusion in the diagnosis, treatment and follow-up process for physicians, patients and their relatives. Almost 20 years after the description of the causal gene defect in the HFE gene, allowing the definite diagnosis of $\mathrm{HH}$, the level of understanding of the medical risk associated with adequate vs. inadequate treatment and follow-up, with regard to the disease-associated complications described above, is virtually unchanged. For instance, there is no clear view on the risk of developing diabetes, once treatment is installed [5], compared to the risk in the general 
population, let alone the risk of developing osteoporosis or hepatocellular carcinoma. One of the factors hampering progress in our understanding of the diseaseassociated risk, in our opinion, is the lack of well-defined standards (i.e. key-interventions, KI's) for screening, treatment and follow-up of $\mathrm{HH}$. International guidelines on $\mathrm{HH}$ exist [6-9] but, as we demonstrated recently, their applicability is limited as they fail to describe how longterm follow-up should be organized and evaluated [10].

To enable treatment evaluation, to support improvement of delivered care and to assist professionals with the delivery of optimal care to their $\mathrm{HH}$ patients, we developed KI's for HH. This study is unique, as the KI's are formulated, starting from recommendations based on the integration of three evidence-based guidelines.

\section{Methods}

A RAND-modified Delphi method was used to develop key-interventions (KIs) related to screening, diagnosis, treatment and follow-up of patients with $\mathrm{HH}$ in five steps, see Fig. 1 [11, 12]. This method combines evidence-based practice with expert opinion by using a multidisciplinary panel in the systematic process of developing KIs [13].

\section{Extraction of recommendations}

References to guidelines on $\mathrm{HH}$ were searched in the Medline database as well as the National Guideline Clearinghouse using following MESH terms: 'haemochromatosis' and 'practice guideline (publication type)' or 'practice guidelines as topic' (June 2013). Only evidencebased guidelines with clearly defined recommendations

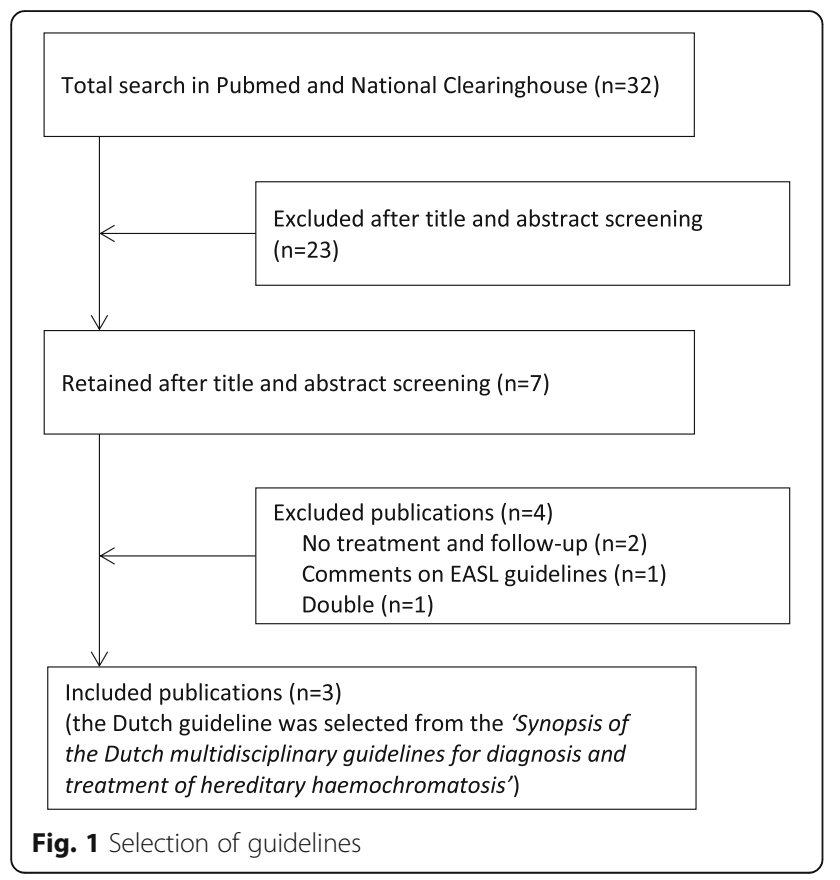

published over the last 10 years were included. If the guideline had been updated, the latest version was used. We included three guidelines from different professional organizations: European Association for the Study of the Liver (EASL), American Association for the Study of Liver Diseases (AASLD) and Netherlands Association of Internal Medicine (NIV), the Netherlands Society of Clinical Chemistry and Laboratory Medicine (NVKC) and Association of Laboratory Physicians (VAL) (DUTCH). These three guidelines on screening, diagnosis and treatment/management of patients with primary $\mathrm{HH}$ were selected because of their clearly defined recommendations [6-10]. The selected guidelines describe evidenced-based recommendations, whether or not in combination with expert opinion recommendations [10]. One researcher (AV) extracted the recommendations from the three guidelines. All recommendations were classified into either 'screening,' diagnosis' or 'treatment/management', together with their Level of Evidence (LoE), where possible. If a recommendation had a slightly different phrasing or if contradictory messages in different guidelines were encountered, this was mentioned in 'comments' (see Additional file 1: Appendix A).

To evaluate these recommendations, a written scoring form was sent to a multidisciplinary team of experts who are involved in the routine care of patients with $\mathrm{HH}$. This multidisciplinary team $(n=17)$ consisted of three hepatogastroenterologists, two rheumatologists, two cardiologists, two endocrinologists, two hematologists, three general practitioners and three nurses.

\section{Written scoring form}

Forty-one recommendations were sent to the multidisciplinary expert team by e-mail (see Additional file 1 : Appendix A). They were asked to score the recommendations on a nine point scale taking into account the following question: 'Is performing this recommendation important for the delivery of high quality of care for patients with HH?' (one point for a bad measure (i.e. no benefit for the patient) and up to nine points for an excellent measure). The multidisciplinary team was asked to take into account 'health gain' (morbidity, mortality, quality of life), 'patient burden' and 'side effects' when scoring the recommendations. When it was impossible for a team member to judge the statement, he/she could mark it as 'impossible to judge'. Panel members were encouraged to add new recommendations or make changes to the existing recommendations. Subsequently, the team members were asked to prioritize the three most relevant recommendations for each domain (screening, diagnosis and treatment/management). The scoring form was filled out by 15 of 17 experts (see Additional file 2: Appendix B). One endocrinologist did not respond despite several reminders. One endocrinologist only suggested two extra 
recommendations regarding diabetes mellitus and did not score the proposed recommendations.

A summary report was drafted, based on the individual results of the written scoring form to facilitate the consensus meeting. This summary report marked the recommendations as having received a 'low, 'uncertain' or 'high' potential to deliver good quality of care in patients with $\mathrm{HH}$ from the experts. Three selection criteria were taken into account, i.e. pre-selection, top-3 percentage and agreement between scorers, which led to a final selection of recommendations.

Pre-selection was summarized into an overall ranking score and median rating. Each participant had to score the top three for screening, diagnosis and treatment/ management. Calculation of this overall rating existed of ranking a recommendation first, second or third with three, two and one point(s) respectively. Taking into account the maximum score (number of panel members who scored this item multiplied by three), the result was converted into percentages. This methodology is described in reference [13].

High potential recommendations were those with a high overall ranking score (top-3 percentage $>20 \%$ ) as well as a high median score $(\geq 8)$. If a recommendation had both a low score on overall ranking (overall ranking of $1-20 \%$ or $<1 \%$ ) and median rating $(<8)$, this recommendation was classified as a recommendation with low potential. Other combinations were classified as recommendations with an uncertain potential (median score $<8$ and top-3 percentage $>20 \%$; overall ranking of $1-20 \%$ and median score of $\geq 8$ ) (Table 1). A cutoff for overall rating of $20 \%$ and a median score of $\geq 8$ was associated with good reproducibility and reliability as well as face validity [13].

\section{Criteria for (dis)agreement}

The evaluation of agreement between the members of the expert panel showed agreement in scoring by $\mathrm{a} \geq$ $70 \%$ scoring in the highest tertile (7-9) (Table 2A). If $\geq$ $30 \%$ of the panel members scored in the lowest tertile (1-3) and $\geq 30 \%$ scored in the highest tertile (7-9) (Table 2B), there was disagreement. Such an item became a discussion point for the consensus meeting. All other combinations resulted in no selection (Table 2C).

Table 1 Key recommendation classification into categories of high, uncertain and low potential according to their overall ranking score and median score

\begin{tabular}{lll}
\hline Overall ranking score & Median score $\geq 8$ & Median score $<8$ \\
\hline Top-3 percentage $>20 \%$ & High potential $(+)$ & Uncertain $(+/-)$ \\
$1-20 \%$ & Uncertain $(+/-)$ & Low potential $(-)$ \\
$<1 \%$ & Low potential $(-)$ & Low potential $(-)$ \\
\hline
\end{tabular}

The items selected for the consensus meeting, were those with a high potential if pre-selection and agreement were positive. A low potential was given to those recommendations which were not pre-selected and showed no agreement or those without agreement and a questionable pre-selection (no selection). All other combinations resulted into recommendations with an uncertain potential (discussion) with regard to delivering high quality of care for patients with $\mathrm{HH}$ (Table 3).

\section{Consensus meeting}

A face-to-face consensus meeting was organized to discuss the recommendations. Consensus was agreed between a hepatogastroentrologist, a hematologist, a cardiologist, a rheumatologist and a specialized nurse. It took $90 \mathrm{~min}$ to discuss the 41 recommendations. An overview of the 'high', 'low' and 'uncertain' potential of each recommendation was given to the panel members (see Additional file 3: Appendix C). Three additional recommendations completed the list for the panel. Recommendations with a high potential were included. There was discussion about the items with an uncertain potential and the extra recommendations for inclusion or rejection. There was also discussion about rejection in case of 'low potential' recommendations. This discussion was moderated by an independent researcher (DS) who did not participate in the scoring of the recommendations.

\section{Final appraisal of recommendations}

The final list of selected recommendations was sent to all scoring panel members after the consensus meeting (see Additional file 4: Appendix D). All 15 panel members agreed with the core set of recommendations.

\section{Development of key-interventions}

The recommendations were subsequently translated into key-interventions for patients with an established diagnosis of $\mathrm{HH}$. These key-interventions were formulated by two researchers (AV and DC).

\section{Results}

Three guidelines met our search criteria: 1 European (EASL), 1 American (AASLD) and 1 guideline from the Netherlands (Dutch) (Fig. 1) [6-9].

At the start, 41 recommendations were extracted from the three guidelines (see Additional file 1: Appendix A). Key recommendations were assigned to three domains: screening (9), diagnosis (7) and treatment/management (25) (Fig. 2). Where possible, the level of evidence was reported (see Additional file 1: Appendix A).

Pre-selection (by priority and median score) and agreement resulted in the inclusion of 28 and exclusion of 13 recommendations, after the consensus meeting. 
Table 2 Examples of agreement and disagreement between panel members

\begin{tabular}{|c|c|c|c|c|c|c|c|c|c|}
\hline$A$ & & rite & & & rile & & & tile & \\
\hline Likert rating scale ${ }^{1}$ & 1 & 2 & 3 & 4 & 5 & 6 & 7 & 8 & 9 \\
\hline No. of rat.ıngs ${ }^{2}$ & 0 & 0 & 0 & 0 & 0 & 1 & 0 & 4 & 10 \\
\hline B & & rtile & & & itile & & & tile & \\
\hline Likert rating $5 \mathrm{cal} \mathrm{e}^{1}$ & 1 & 2 & $\exists$ & 4 & 5 & 6 & 7 & 8 & 9 \\
\hline No. of ratings ${ }^{2}$ & 1 & 1 & 1 & 1 & 2 & 2 & 2 & 2 & 0 \\
\hline C & & rtile & & & itile & & & til & \\
\hline Likert rating scale ${ }^{1}$ & 1 & 2 & 3 & 4 & 5 & 6 & 7 & B & 9 \\
\hline No. of ratings ${ }^{2}$ & 0 & 1 & 1 & 0 & 2 & 2 & 3 & 1 & 2 \\
\hline
\end{tabular}

A: Agreement (+: selection), B: Discussion Point (+/-), C: Disagreement (-: no selection)

The three suggested additional recommendations were also excluded: two were related to screening of $\mathrm{HH}$ in patients with diabetes mellitus, the third was related to age restrictions to start phlebotomies. The panel decided that a general recommendation about age restriction could not be given, but that on the contrary there was a need for an evaluation of each individual case. From the total of 28 included recommendations, eight were merged into four recommendations as their content overlapped. Finally, 15 of the 24 recommendations were transformed into KI's (Table 4). Nine initial recommendations were excluded because they could not be transformed into measurable indicators or were related to screening for $\mathrm{HH}$, while our study aims at patients with

Table 3 Selection of items after scoring

\begin{tabular}{lll}
\hline Preselection & Agreement & Final label \\
\hline+ & - & Discussion \\
- & - & No selection \\
- & + & Discussion \\
+ & + & Selection \\
$+/-$ & + & Discussion \\
$+/-$ & - & No selection \\
$+/-$ & $+/-$ & Discussion \\
+ & $+/-$ & Discussion \\
- & $+/-$ & No selection \\
\hline
\end{tabular}

an established diagnosis of HH. For instance: 'HFE testing should be considered in patients with type 1 diabetes in case of abnormal iron parameters' (Table 5).

\section{Screening}

'First-degree relatives of HH patients must be screened' is highlighted as the most important recommendation in the 'screening' section and the only one which could be transferred into a KI. All three guidelines (EASL, AASLD and Dutch) promote screening of first-degree relatives in patients with $\mathrm{HH}$ [6-8]. Other recommendations were excluded (i.e. screening of patient populations, presenting with e.g. chronic hepatitis or other symptoms possibly related to $\mathrm{HH}$ ) since they were not related to $\mathrm{HH}$ patients and are therefore outside the scope of our study.

\section{Treatment/management}

The treatment and management section was subdivided into 'phlebotomy', 'examinations', 'general issues' and 'diet/lifestyle'.

\section{Phlebotomy}

All recommendations regarding phlebotomy were transferred into KI's. Two of them were merged into one KI. There was consensus between the three guidelines, i.e. 'HH patients with raised ferritin levels must start treatment with (bi)weekly phlebotomy (removing 400-500 ml 


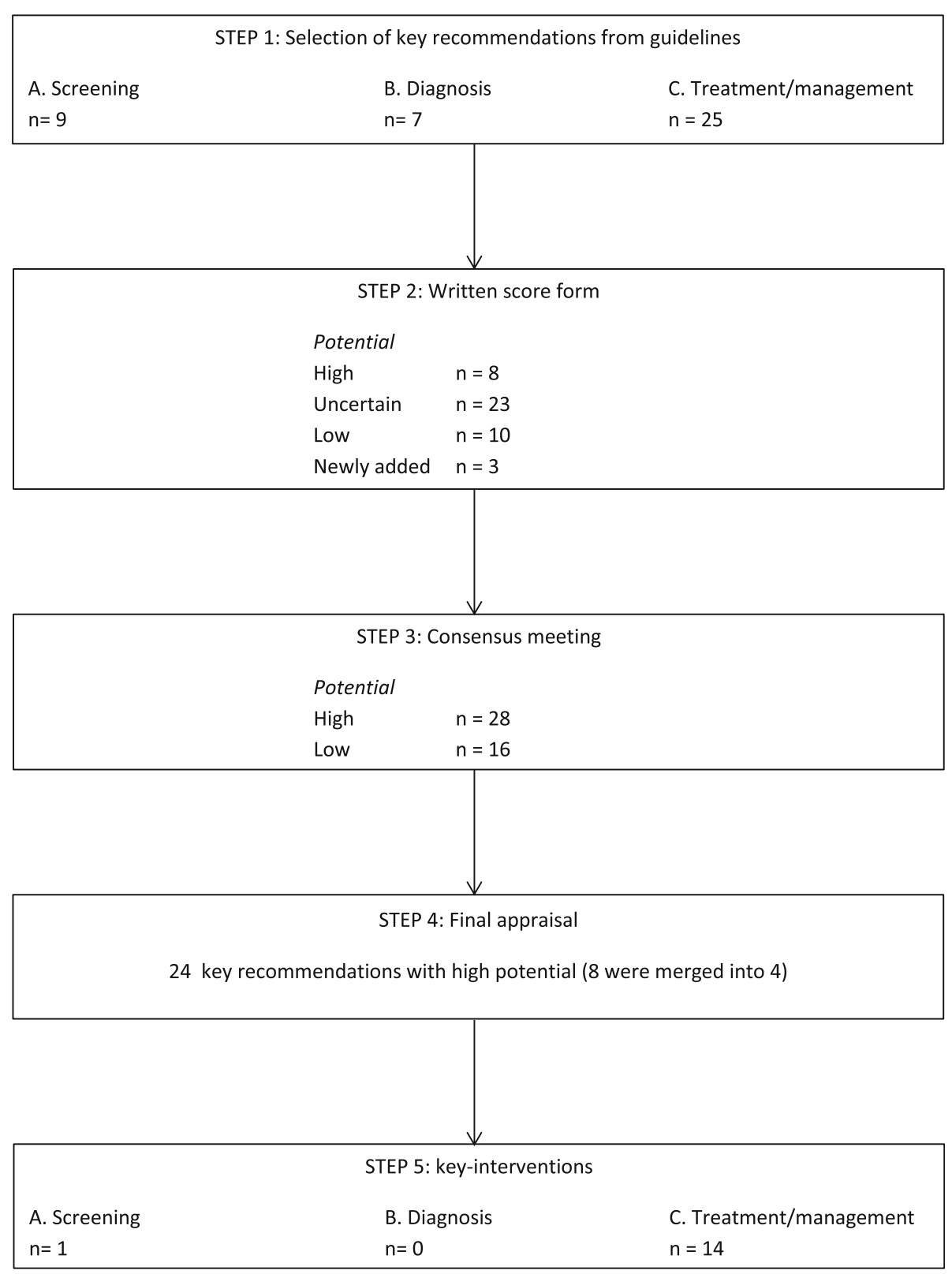

Fig. 2 Selection of key-interventions

of blood)'. The AASLD clearly promotes a target level of serum ferritin (SF) between 50 and $100 \mu \mathrm{g} / \mathrm{L}$ [7]. On the other hand, the Dutch guideline requires a target SF level under the upper limit of normal [8]. As important differences between the different laboratories reporting transferrin saturation (TS) and SF occur, the expert panel agreed with targeting a SF level between 50 and $100 \mu \mathrm{g} / \mathrm{L}$, to have a clear cut-off point. There was consensus between the three guidelines as well as between the experts, that patients with organ damage should undergo phlebotomies in case of iron overload.

\section{Examinations}

The EASL guideline describes that patients must undergo examinations (transient elastography ((fibroscan), a non-invasive liver stiffness measurement (Echosens, France)), liver biopsy) in order to detect liver damage early [6]. During the consensus meeting, two recommendations were merged into one, which was transformed into the following KI 'Screening for liver fibrosis or cirrhosis in $\mathrm{HH}$ patients must be performed and can be performed using either transient elastography or biopsy'. 
Table 4 Key-interventions to treat patients with $\mathrm{HH}$

Screening
1. First-degree relatives of HH patients must be screened.
Treatment/management
Phlebotomy
2. HH patients with raised ferritin levels must start treatment with
(bi)weekly phlebotomy (removing $400-500$ ml of blood).
3. The ferritin target level for HH patients 'on treatment' is between
50-100 $\mu$ g/L.
4. HH patients without indicators of significant liver disease (AST and/or
ALT elevation), but with elevated ferritin, must also proceed to
phlebotomies.
5. HH patients with advanced liver fibrosis or cirrhosis can safely undergo
phlebotomy and must also be treated.
6. HH patients undergoing phlebotomies must be advised to take
adequate hydration before and after treatment and avoid vigorous
physical activity for 24 h after treatment.
Examinations
7. Screening for liver fibrosis or cirrhosis in HH patients must be performed
and can be performed using either transient elastography or biopsy.
General issues
8. HH patients without evidence of iron overload must be monitored
annually and treated when the ferritin rises above normal.
9. HH patients must be immunized against hepatitis A and B.
10. HH patients with cirrhosis must receive yearly influenza and 5-yearly
pneumococci vaccination.
11. HH patients with cirrhosis must be screened every 6 months for
focal liver lesions (by ultrasound and serum alpha fetoprotein).
12. HH patients must be assessed and managed for complications (liver
disease, diabetes mellitus, joint diseases, endocrine deficiency
(hypothyroidism), cardiac disease, porphyria cutanea tarda and
osteoporosis).
13. Fasting glycemia and/or HbA1c must be measured yearly in HH patients.
14. HH patients who have complaints compatible with osteoarthritis,
Diet/lifestyle
15. HH patients in the iron depletion phase must avoid the intake of alcohol.
andergo physical and radiological evaluation.

\section{General issues}

In contrast to the Dutch guideline, which requires monitoring C282Y homozygotes without iron overload every 3 years, the EASL as well as the AASLD require annual monitoring [6-8]. Considering that annual monitoring is easier to organize for the health care providers, the expert panel decided that patients should monitor their SF yearly (although this is more costly) and start treatment when the ferritin level rises above the upper limit of the reference range.

Immunization against hepatitis A (HAV) and B $(\mathrm{HBV})$ is an important issue in patients with $\mathrm{HH}$ because patients with a chronic liver disease are at higher risk to develop complications of HAV and HBV [14]. In addition, patients with cirrhosis must be immunized against influenza yearly and pneumococci every 5 years. All three recommendations are supported by the EASL and were transformed into a KI.

During the consensus meeting, the recommendation to screen cirrhotic patients for focal liver lesions by ultrasound
Table 5 Recommendations to screen for $\mathrm{HH}$ in other patient populations

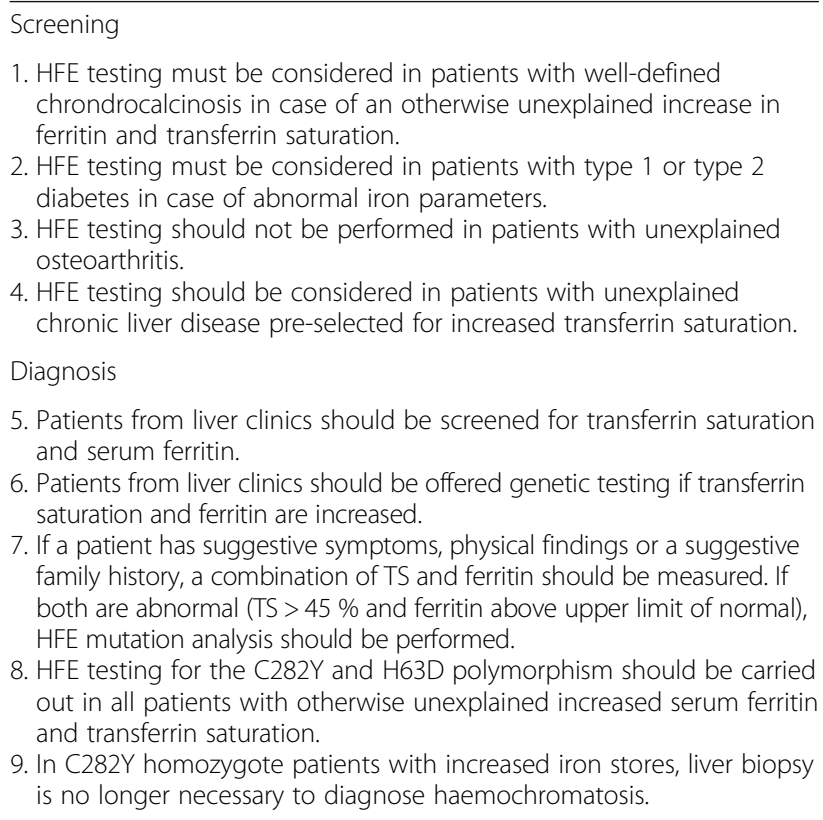

1. HFE testing must be considered in patients with well-defined chrondrocalcinosis in case of an otherwise unexplained increase in ferritin and transferrin saturation.

2. HFE testing must be considered in patients with type 1 or type 2 diabetes in case of abnormal iron parameters.

3. HFE testing should not be performed in patients with unexplained osteoarthritis.

4. HFE testing should be considered in patients with unexplained chronic liver disease pre-selected for increased transferrin saturation.

Diagnosis

5. Patients from liver clinics should be screened for transferrin saturation and serum ferritin.

6. Patients from liver clinics should be offered genetic testing if transferrin saturation and ferritin are increased.

7. If a patient has suggestive symptoms, physical findings or a suggestive family history, a combination of TS and ferritin should be measured. If both are abnormal (TS > $45 \%$ and ferritin above upper limit of normal), HFE mutation analysis should be performed.

8. HFE testing for the C282Y and H63D polymorphism should be carried out in all patients with otherwise unexplained increased serum ferritin and transferrin saturation.

9. In C282Y homozygote patients with increased iron stores, liver biopsy is no longer necessary to diagnose haemochromatosis.

as well as measurement of serum alpha fetoprotein every 6 months, was not challenged and supported by the European as well as the Dutch guidelines [6-8].

Patients with $\mathrm{HH}$ can develop a variety of medical problems, including liver disease, diabetes mellitus, joint disease, endocrine deficiencies (hypothyroidism, hypogonadism), cardiac disease, porphyria cutanea tarda and osteoporosis [2-4]. Assessment and eventual management of these problems are necessary in order to prevent patients developing worse or additional complications. Considering this, the expert panel agreed that patients should have their fasting glycemia and/or HbA1c checked yearly, to detect diabetes mellitus early. Furthermore, the following EASL recommendation: 'physical and radiological evaluation is necessary to evaluate possible arthralgia and arthritis' was changed during the consensus meeting into 'HH patients who have complaints compatible with osteoarthritis, must undergo physical and radiological evaluation'. All these recommendations were transformed into KI's (Table 4).

\section{Diet/lifestyle}

In theory, additional iron taken up via the diet or via supplements can be removed via phlebotomy. In general, however, most physicians will advise their patients to avoid extra iron uptake from the diet, by avoiding Vitamin C supplements taken with meals, as well as the intake of iron supplements. The expert panel considered that every person, whatever his/her health status is, needs a healthy diet. Therefore we only selected the recommendation that patients at start of treatment must be advised to avoid the intake of alcohol. 


\section{Recommendations that were not selected}

Nine out of 24 recommendations which were highlighted as recommendations to deliver good quality of care were not selected to be transformed into KI's. Four were derived from the screening section of the recommendations. Secondly, five diagnostic recommendations could not be transformed in quantifiable KI's (Table 5). The nine recommendations that did not qualify as KI's, were all valid recommendations but not related to the measurement of the quality of treatment or management of patients who are already diagnosed with $\mathrm{HH}$.

\section{Discussion}

This is the first study describing the development of 15 guideline-based KI's, derived from three evidence-based guidelines on $\mathrm{HH}$, by a multidisciplinary expert panel. The KI's relate to screening, diagnosis and treatment/ management of $\mathrm{HH}$. The final list of $15 \mathrm{KI}$ 's provides professionals with parameters to measure and follow-up quality of care in patients with $\mathrm{HH}$, thereby preventing the development of serious, potentially fatal complications in this patient population.

The uniqueness of this study lies in the fact that we developed KI's by starting from three evidence-based guidelines, and followed by applying a RAND modified Delphi method to develop consensus. Evidence-based KI's are the active ingredients in care pathways (CPs) $[15,16]$. CPs aim to improve patient processes and outcomes by (re)organizing care processes. The European Pathway Association defines a $\mathrm{CP}$ as "a complex intervention for the mutual decision making and organization of care for a well-defined group of patients during a well-defined period" [17]. The integration of CPs in a well-defined group of patients was already described in several patient settings, i.e. Chronic Obstructive Pulmonary Disease, Proximal Femur Fracture, patients with stroke [18-20]. This resulted in better interprofessional teamwork, a higher level of organized care and a lower risk of burnout in the case of CPs in acute health care teams [19]. By using CPs, the care delivered to stroke patients resulted in more effective treatment and better use of organized care [20]. Clinical content of an evidence-based CP can be created through the eightstep method developed by Lodewijckx et al. [18]. Our selected KI's will indeed be used to create a CP by using hospital-related indicators,and including patient and organizational indicators will also be considered.

Earlier research shows that outcomes are influenced by the composition of the expert panel [21]. The strength of our study is that 15 independent clinical experts scored the 41 recommendations. The experts were selected from six different hospitals (tertiary university hospitals, as well as regional hospitals), from primary care and from two universities in Belgium. The appraisers are active in 7 different medical specialties (see Additional file 2: Appendix B). The consensus meeting was headed by an independent expert (DS) in $\mathrm{HH}$, from the Netherlands. A limiting factor of our study is that only 5 experts participated in the consensus meeting, for logistical reasons and that no patient representatives were involved. Nevertheless, only the opinion of a general practitioner was absent from the consensus meeting and all 15 appraisers agreed with the final list of 15 recommendations, drafted after the consensus meeting.

The resulting set of KI's, which is a combination of evidence and expert opinion, is developed to be applicable in different health care settings: hospitals as well as primary care settings. For this reason, the set of KI's can be used by different health care professionals, i.e. physicians (hepatogastroenterologists, hematologists, rheumatologists, general practitioners, ...) as well as advanced nurse practitioners [22].

Although we did not probe for prioritization between the recommendations, at the consensus meeting, the most important recommendation is clearly that "patients with raised ferritin levels should start treatment with (bi) weekly phlebotomy" (recommendation nr. 2), which is also highlighted in the questionnaire round (see Additional file 3: Appendix $\mathrm{C}$ ) and by the three guidelines [6-8]. No recommendation about apheresis in $\mathrm{HH}$ patients is included, since this was not described in the referring guidelines. The goal of treatment is to prevent $\mathrm{HH}$ patients from developing complications of the disease and, therefore, patients should be assessed for complications and those need to be managed in case they are present. A longitudinal follow-up, applying the KIs we propose here, may in addition finally reveal whether patients with $\mathrm{HH}$ - treated adequately - still have an increased risk to develop diabetes.

As described in Additional file 1: Appendix A, there is a difference in wording of the recommendations between the three selected guidelines, but there are no important contradictions. During the consensus meeting, some recommendations were merged or adapted in view of the development of KI's (Additional file 4: Appendix D).

Limitations to this study are that patient representatives are not included in the expert panel notwithstanding the fact that $\mathrm{HH}$ patients in general are able to assume control of their own care process. We are therefore planning further qualitative research on how patients experience the quality of the delivered care by face-to-face interviews, questionnaires and focus group interviews. We are particularly interested in further care coordination in primary care and self-management by patients, since integrated patient care is becoming more and more important [23].

The final list of 15 recommendations forms the basis for the measurement of the quality of the usual clinical 
care and allows to objectively quantify the effects of adaptations to the care path of the $\mathrm{HH}$ patient population. A practice test to assess the measurability of the KI's is planned, as well as a study demonstrating improvement of outcome, when the KI's are applied.

\section{Conclusion}

$\mathrm{HH}$ is a common disorder with potentially life-threatening complications, if left untreated or not treated properly before irreversible organ damage occurs. To optimize the care for $\mathrm{HH}$ patients, we developed a set of 15 measurable clinical KI's. This set of standardized KI's now allows us to measure, adapt and deliver high quality of care for patients with $\mathrm{HH}$ in the hospital setting as well as in primary care.

\section{Additional files}

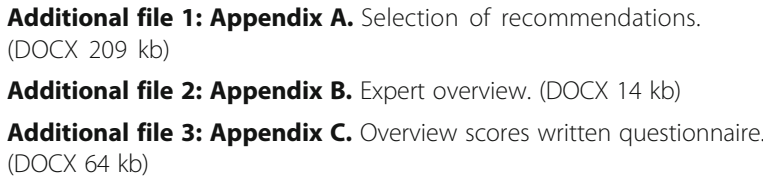

Additional file 1: Appendix A. Selection of recommendations. (DOCX 209 kb)

Additional file 2: Appendix B. Expert overview. (DOCX $14 \mathrm{~kb}$ ) Additional file 3: Appendix C. Overview scores written questionnaire. (DOCX 64 kb)

Additional file 4: Appendix D. Selected recommendations. (DOCX 18 kb)

\section{Abbreviations}

AASLD: American Association for the Study of Liver Diseases; CP: Care Pathway; DUTCH: Dutch guidelines for hereditary haemochromatosis; EASL: European Association for the Study of the Liver; HAV: Hepatitis A virus; HBV: Hepatitis B virus; $\mathrm{HH}$ : Hereditary haemochromatosis; Kl: Key-intervention; LoE: Level of Evidence; SF: Serum ferritin; TS: Transferrin saturation

\section{The haemochromatosis working group:}

Rene Westhovens scored all recommendations and approved the final version. Johan Van Cleemput scored all recommendations and approved the final version. Vincent Maertens scored all recommendations and approved the final version. Hans Van Vlierberghe scored all recommendations and approved the final version. Rudy Harlet scored all recommendations and approved the final version. Patrick Verschueren scored all recommendations and approved the final version. Walter Droogne scored all recommendations and approved the final version. Koen Theunissen scored all recommendations and approved the final version. Neree Claes scored all recommendations and approved the final version. Hannelore Van Droogenbroek scored all recommendations and approved the final version.

Patrik Vankrunkelsven scored all recommendations and approved the final version Chantal Mathieu scored recommendations about endocrinology and approved the final version.

Sabien Severi scored all recommendations and approved the final version. Ilse Scherens scored all recommendations and approved the final version. Sandra Rottiers scored all recommendations and approved the final version. Prof. R. Westhovens (UH Leuven), prof. J. Van Cleemput (UH Leuven), dr. V. Maertens (Imelda Hospital Bonheiden), Prof. H. Van Vlierberghe (UH Ghent), dr. R. Harlet (AH Turnhout), prof. P. Verschueren (UH Leuven), dr. W. Droogne (UH Leuven), dr. K. Theunissen (Jessa-Hospital Hasselt), Prof. N. Claes (UHasselt), dr. H. Van Droogenbroek (General practitioner), prof. P. Vankrunkelsven (University of Leuven), prof. C. Mathieu (UH Leuven), S. Severi (UH Leuven), Scherens (UH Leuven), S. Rottiers (UH Leuven).

\section{Funding}

None

\section{Availability of data and materials}

All data generated or analysed during this study are included in this published article and its supplementary information files:

\section{Authors' contributions}

AV designed the study, did the acquisition, analysis and interpretation of data and wrote the article. HW designed the study, scored the recommendations, was involved in the interpretation, revised the article critically and approved the final version. $\mathrm{KV}$ was involved in the interpretation of data, revised the article critically and approved the final version. DS was involved in the interpretation of data, revised the article critically and approved the final version. BA designed the study, scored the recommendations, was involved in the interpretation, revised the article critically and approved the final version. DC designed the study, scored the recommendations, was involved in the interpretation, revised the article critically and approved the final version.

\section{Authors' information}

David Cassiman is a fundamental-clinical researcher for FWO-Vlaanderen.

\section{Competing interests}

The authors declare that they have no competing interests.

\section{Consent for publication}

Not applicable.

\section{Ethics approval and consent to participate}

This study was approved by the ethical committee of the University Hospitals Leuven, Gasthuisberg, Belgium (B322201420053, ML9999 DGA). There were no participants involved, only experts who are subject to freedom of speech and opinion, there was verbal consent. The participation of the experts was voluntary. The participants were informed that their responses would be used for research, they all approved the final Kls list and are referred to as 'the haemochromatosis working group'. All participants were medical professionals, there were no patients involved.

\section{Author details}

${ }^{1}$ Department of Hepatology and Metabolic Center, University Hospital Gasthuisberg, Herestraat 49, 3000 Leuven, Belgium. ${ }^{2}$ Scientific Institute for Quality of Healthcare, Nijmegen Centre for Evidence Based Practice, Radboud University Medical Centre, Nijmegen, The Netherlands. ${ }^{3}$ Department of Public Health and Primary Care, Health Services Research Group, KU Leuven, Leuven, Belgium. ${ }^{4}$ Department of Laboratory Medicine, Laboratory of Genetic Endocrine and Metabolic diseases, Radboud University Medical Centre, Nijmegen, The Netherlands. ${ }^{5}$ Academic Center for General Practice, KU Leuven, Leuven, Belgium.

Received: 15 July 2015 Accepted: 8 October 2016

Published online: 13 October 2016

References

1. Feder JN, Gnirke A, Thomas W, Tsuchihashi Z, Ruddy DA, Basava A, et al. A novel MHC class I-like gene is mutated in patients with hereditary haemochromatosis. Nat Genet. 1996;13(4):399-408.

2. Pietrangelo A. Hereditary hemochromatosis: pathogenesis, diagnosis, and treatment. Gastroenterology. 2010;139(2):393-408.

3. Niederau C, Fischer R, Pürschel A, Stremmel W, Häussinger D, Strohmeyer G. Long-term survival in patients with hereditary hemochromatosis. Gastroenterology. 1996;110(4):1107-19.

4. van Bokhoven MA, van Deursen CT, Swinkels DW. Diagnosis and management of hereditary haemochromatosis. BMJ (Clinical research ed) 2011;342:C7251.

5. Mitchell TC, McClain DA. Diabetes and Hemochromatosis. Curr Diab Rep. 2014;14(5):488. doi:10.1007/s11892-014-0488-y.

6. European Association For The Study Of The Liver. EASL clinical practice guidelines for HFE hemochromatosis. J Hepatol. 2010;53(1):3-22.

7. Bacon BR, Adams PC, Kowdley KV, et al. Diagnosis and management of hemochromatosis: 2011 Practice Guideline by the American Association for the Study of Liver Diseases. Hepatology. 2011;54(1):328-43.

8. Vereniging Nederlandse Internisten. Richtlijn Hereditaire Hemochromatose Diagnostiek en behandeling van hereditaire hemochromatose. 2007. [http:// www.internisten.nl/uploads/AL/FJ/ALFJChidQ2OgF7uax8yh1w/richtlijn 2007_Hemochromatose.pdf] (Accessed 20 June 2013). 
9. Swinkels DW, Jorna AT, Raymakers RA. Synopsis of the Dutch multidisciplinary guideline for the diagnosis and treatment of hereditary haemochromatosis. Neth J Med. 2007;65(11):452-5.

10. Vanclooster A, Cassiman D, Van Steenbergen W, Swinkels DW, Janssen MCH, Drenth JPH, Aertgeerts B, Wollersheim $\mathrm{H}$. The quality of hereditary haemochromatosis guidelines: a comparative analysis. Clin Res in Hepatol Gastroenterol. 2015;39(2):205-14.

11. Campbell SM, Braspenning J, Hutchinson A, Marshall MN. Research methods used in developing and applying quality indicators in primary care. BMJ (Clinical research ed) 2003;326:816-9.

12. Uphoff EP, Wennekes L, Punt CJ, Grol RP, Wollersheim HC, Hermens RP, et al. Development of generic quality indicators for patient-centered cancer care by using a RAND modified Delphi method. Cancer Nurs. 2012:35(1):29-37.

13. Fitch $K$, Bernstein $S J$, Aguilar MS, Burnand B, LaCalle JR, L zaro P, et al. The RAND/UCLA Appropriateness Method User's Manual. Arlington: RAND; 2001. p. 123.

14. Loulergue P, Pol S, Mallet V, Sogni P, Launay O, Gevaccim Group. Why actively promote vaccination in patients with cirrhosis? J Clin Virol. 2009 46(3):206-9

15. Vanhaecht K, De Witte K, Sermeus W. The care process organization triangle: a framework to better understand how clinical pathways work. Int J Care Pathw. 2007:11:1-8.

16. Vanhaecht K, Sermeus W, Peers J, Lodewijckx C, Deneckere S, Leigheb F, et al. The impact of care pathways for the exacerbation of chronic obstructive pulmonary disease: rationale and design of a cluster randomized controlled trial. Trials. 2010;11:111

17. Vanhaecht K, De Witte K, Sermeus W. General introduction. In the Impact of clinical pathways on the organization of care processes. ACCO, Leuven, pp. 1-18.

18. Lodewijckx C, Decramer M, Sermeus W, Panella M, Deneckere S, Vanhaecht K. Eight-step method to build the clinical content of an evidence-based care pathways: the case for COPD exacerbation. Trials. 2012;13:229.

19. Deneckere S, Euwema M, Lodewijckx C, Panella M, Mutsvari T, Sermeus W, et al. Better interprofessional teamwork, higher level of organized care, and lower risk of burnout in acute health care teams using care pathways. A cluster randomized controlled trial. Med Care. 2013:51:99-107.

20. Panella M, Marchisio S, Brambilla R, Vanhaecht K, Di Stanislao F. A cluster randomized trial to assess the effect of clinical pathways for patients with stroke: results of the clinical pathways for effective and appropriate care study. BMC Med. 2012;10:71.

21. Campbell SM, Hann M, Roland MO, Quayle JA, Shekelle PG. The effect of panel membership and feedback on ratings in a two-round Delphi survey: results of a randomized controlled trial. Med Care. 1999;37(9):964-8.

22. Emanuele D, Tuason I, Edwards QT. HFE-associated hereditary hemochromatosis: overview of genetics and clinical implications for nurse practitioners in primary care settings. J Am Assoc Nurse Pract. 2014;26(3):113-22.

23. Singer SJ, Burgers J, Friedberg M, Rosenthal MB, Leape L, Schneider E. Defining and measuring integrated patient care: promoting the next frontier in health care delivery. Med Care Res Rev. 2011;68(1):112-27.

\section{Submit your next manuscript to BioMed Central and we will help you at every step:}

- We accept pre-submission inquiries

- Our selector tool helps you to find the most relevant journal

- We provide round the clock customer support

- Convenient online submission

- Thorough peer review

- Inclusion in PubMed and all major indexing services

- Maximum visibility for your research

Submit your manuscript at www.biomedcentral.com/submit

) Biomed Central 